\title{
TEMPO DE VENTILAÇÃO MECÂNICA E DESENVOLVIMENTO DE DISPLASIA BRONCOPULMONAR
}

\author{
Ana Damaris Gonzaga*, Bettina B. Duque figueira, José Marconi A. Sousa, Werther Brunow de Carvalho \\ Trabalho realizado na Disciplina de Pneumologia da Universidade Federal de São Paulo / Escola Paulista de Medicina
}

\section{*Correspondência:}

Rua Herculano de Freitas,

47, apto 74, Bela Vista

São Paulo/SP

Cep: 01308-020

Tels: (11) 3259-5461 / (11)

8185-9920

anadamarisg@yahoo.com.br

\section{RESUMO}

OBjetivo. Verificar a associação entre o tempo de uso da ventilação mecânica e o desenvolvimento de displasia broncopulmonar em recém-nascidos com peso de nascimento $\leq 1500 \mathrm{~g}$.

Métodos. Pesquisa retrospectiva em recém-nascidos com peso de nascimento $\leq 1500 \mathrm{~g}$ que utilizaram ventilação mecânica. Foram excluídos as malformações congênitas maiores, transferências e óbitos antes do $28^{\circ}$ dia de vida. Foram analisados três grupos de acordo com o tempo de uso da ventilação mecânica: I a 7 dias, 8 a I 4 dias e $\geq 15$ dias. Foi calculada a razão de chance para o desenvolvimento de displasia broncopulmonar em cada período de utilizaçãa da ventilação mecânica.

Resultados. Dos 216 prontuários avaliados, 121 preencheram os critérios de inclusão. As médias do peso de nascimento e idade gestacional foram de 1 199,8 g e 31,8 semanas. No período de I a 7 dias de uso da ventilação mecânica, 15,5\% dos recém-nascidos evoluíram com displasia broncopulmonar; no período de 8 a 14 dias, 60\%; e no período $\geq 15$ dias, 88,2\%; com razão de chance de 0,16,11,25 e 16,36, respectivamente.

Conclusão. A possibilidade de um recém-nascido com peso de nascimento $\leq 1500 \mathrm{~g}$ desenvolver displasia broncopulmonar foi II vezes maior naqueles que permaneceram em ventilação mecânica por até 14 dias e esta chance aumentou ainda mais nos que foram ventilados por mais de 15 dias, devendo a equipe que presta atendimento ao paciente de alto risco estar empenhada na extubação dos recém-nascidos ainda na primeira semana de vida.

UnITERMos: Displasia broncopulmonar. Recém-nascido. Muito baixo peso. Ventilação mecânica.

\section{INTRODUÇÃO}

O avanço na medicina perinatal, incluindo a introdução do surfactante exógeno, o uso do corticóide pré-natal e a melhora nos cuidados intensivos neonatais, tem se relacionado a um aumento importante na sobrevida de recém-nascidos prematuros de muito baixo peso. Entretanto, a esse benefício se contrapõe o aumento concomitante da incidência de displasia broncopulmonar (DBP), permanecendo essa patologia como a maior complicação nos prematuros'.

O uso da ventilação pulmonar mecânica tem sido identificado como um dos principais fatores de lesão pulmonar em prematuros. Apesar do seu efeito potencial salvador de vidas, esse recurso apresenta vários riscos e complicações inerentes a seu uso. A ruptura das paredes do espaço aéreo - barotrauma - é a lesão mais freqüente. Nessa situação, ocorre acúmulo de ar extra-alveolar com manifestações clínicas graves, das quais o pneumotórax hipertensivo é a mais deletéria. Além dos eventos macroscópicos do barotrauma, alterações mais sutis, fisiológicas, morfológicas e celulares têm sido relatadas ${ }^{2-4}$. Essa forma de lesão tem sido objeto de constante preocupação das equipes que utilizam a ventilação pulmonar mecânica em neonatologia.

Estudos realizados com pacientes ventilados mecanicamente demonstram alterações do líquido extravascular pulmonar, na permeabilidade capilar, na produção de mediadores inflamatórios e no desenvolvimento de necrose celular. A lesão pulmonar provocada pela ventilação pulmonar mecânica pode gerar alterações anatomopatológicas que não diferem, fundamentalmente, da lesão pulmonar difusa que se observa em pacientes com síndrome do desconforto respiratório agudo. Dessa forma, a lesão pulmonar induzida pela ventilação pulmonar mecânica pode ser indistinguível do processo patológico inicial. Essas observações têm levado à utilização de estratégias ventilatórias protetoras, na tentativa de minimizar os efeitos lesivos da ventilação pulmonar mecânica ${ }^{5}$.

Embora a lesão pulmonar induzida pela ventilação pulmonar mecânica venha sendo amplamente estudada, uma melhor definição sobre o momento exato em que o seu emprego é crucial para o desenvolvimento da DBP nos ajudará na prevenção dessa patologia, que freqüentemente ocasiona internações prolongadas com sérias conseqüências para o prematuro, sua família e a sociedade.

Dessa forma, o objetivo principal desta pesquisa foi verificar a associação entre o tempo de uso da ventilação pulmonar mecânica e o desenvolvimento de DBP em recém-nascidos com peso de nascimento $\leq 1500 \mathrm{~g}$.

\section{Métodos}

Estudo retrospectivo, realizado em uma instituição hospitalar pública do município de São Paulo, no período de $1^{\circ}$ de janeiro de 2001 a 31 de dezembro de 2002, em que foram incluídos os recém-nascidos 
Tabela I - Características da população estudada de acordo com o tempo de uso da ventilação pulmonar mecânica

\begin{tabular}{|c|c|c|c|c|}
\hline \multirow[t]{3}{*}{ Tempo de uso de VPM } & \multirow[t]{2}{*}{ Variáveis } & \multicolumn{2}{|c|}{ DBP } & \multirow[t]{2}{*}{$p$} \\
\hline & & Sim & Não & \\
\hline & & Média \pm DP & Média $\pm \mathrm{DP}$ & \\
\hline I a 7 dias & $\begin{array}{l}\text { PN } \\
\text { IG } \\
\text { CRIB }\end{array}$ & $\begin{array}{c}11|60 \pm| 5 \mid, 4 \\
32,0 \pm 1,5 \\
3,0 \pm 2,0\end{array}$ & $\begin{array}{c}1273 \pm 157,7 \\
32,1 \pm 1,3 \\
2,6 \pm 1,8\end{array}$ & $\begin{array}{l}0,088 \\
0,922 \\
0,567\end{array}$ \\
\hline \multirow{2}{*}{ 8a 14 dias } & PN & $1240 \pm 224,4$ & $1288,0 \pm 123,8$ & 0,545 \\
\hline & $\begin{array}{c}\text { IG } \\
\text { CRIB }\end{array}$ & $\begin{array}{l}31,5 \pm 1,5 \\
3,8 \pm 2.6\end{array}$ & $\begin{array}{c}31,3 \pm 2,0 \\
4.1 \pm 2,5\end{array}$ & $\begin{array}{l}0,836 \\
0,778\end{array}$ \\
\hline \multirow[t]{3}{*}{$\geq 15$ dias } & PN & $10 \mid 1,0 \pm 170,7$ & $|320,8 \pm| 79, \mid$ & 0,001 \\
\hline & IG & $30,4 \pm 2,2$ & $32,9 \pm 2,0$ & 0,002 \\
\hline & CRIB & $5,6 \pm 3,1$ & $4,5 \pm 2,9$ & 0,316 \\
\hline
\end{tabular}

VPM: ventilação pulmonar mecânica; DBP: displasia broncopulmonar; PN: peso de nascimento; IG: idade gestacional; CRIB: clinical risk index for babies

Tabela 2 - Ocorrência e razão de chance de displasia broncopulmonar de acordo com o tempo de utilização da ventilação pulmonar mecânica

\begin{tabular}{|c|c|c|c|}
\hline Tempo de uso de VPM & $\begin{array}{c}\text { DBP } \\
\mathrm{n} / \mathrm{n} \text { total }(\%)\end{array}$ & Razão de chance & $\begin{array}{l}\text { Intervalo de confiança } \\
\text { (IC 95\%) }\end{array}$ \\
\hline I a 7 dias & $7 / 45(15,5)$ & 0,16 & $0,068-0,402$ \\
\hline 8 a 14 dias & $15 / 25(60,0)$ & 11,25 & $3,50-36,20$ \\
\hline$\geq 15$ dias & $45 / 51(88,2)$ & 16,36 & $5,68-52,63$ \\
\hline
\end{tabular}

VPM: ventilação pulmonar mecânica; DBP: displasia broncopulmonar

com peso de nascimento $\leq 1500 \mathrm{~g}$ que utilizaram ventilação pulmonar mecânica. Foram excluídos os recém-nascidos portadores de malformação congênita maior (que apresentavam maior risco de morrer em decorrência da alteração) ${ }^{6}$, transferências para outros serviços e óbitos antes do $28^{\circ}$ dia de vida.

O estudo foi aprovado pelos Comitês de Ética em Pesquisa da Unifesp/EPM e do Hospital e Maternidade Leonor Mendes de Barros.

O levantamento de dados foi realizado por meio da revisão de 216 prontuários, sendo que estes foram transcritos para um formulário previamente validado, desenvolvido especialmente para a pesquisa. A validação do formulário foi realizada por meio de um estudo piloto contendo I 5 pacientes. As variáveis analisadas foram idade gestacional, peso de nascimento, escore prognóstico CRIB (Clinical risk index for babies $)^{7}$, tempo de uso de ventilação pulmonar mecânica, presença de DBP e condição de alta.

Foi considerada DBP o uso contínuo de oxigênio até o $28^{\circ}$ dia de vida ${ }^{8}$. O tempo de ventilação pulmonar mecânica foi mensurado em dias, sendo considerados dias completos aqueles em que o uso foi superior a 12 horas e desconsiderados quando utilizada por um período menor que esse. Foram considerados apenas os casos de utilização contínua da ventilação pulmonar mecânica, sendo analisados três grupos de acordo com o tempo de uso de ventilação pulmonar mecânica: I a 7 dias, 8 a $\mid 4$ dias e $\geq 15$ dias.

Os aparelhos de ventilação pulmonar mecânica utilizados foram da marca Inter $3 \circledR$ (Intermed), na modalidade ventilação mandatóriointermitente, com fluxo contínuo, ciclados a tempo e limitados a pressão.
As variáveis contínuas com distribuição normal foram comparadas pelo teste $t$ de Student e as variáveis categóricas pelo teste Qui-quadrado. Para verificar a associação entre DBP e o uso de ventilação pulmonar mecânica, foi calculada a razão de chance em cada período de utilização desse recurso. Valores de $p<0,05$ foram considerados significantes.

\section{Resultados}

Nasceram, no período de estudo, 216 bebês com peso $\leq 1500 \mathrm{~g}$. Desses, 50 evoluíram para óbito antes do $28^{\circ}$ dia de vida, 16 foram transferidos para outros hospitais e 29 não utilizaram ventilação pulmonar mecânica, restando $12 \mid$ recém-nascidos que compuseram a população de estudo. A média do peso de nascimento foi de $1199,8 \pm 207,8 \mathrm{~g}$, da idade gestacional de $31,8 \pm 2,2$ semanas e do escore prognóstico CRIB de 4, $10 \pm 1,34$. Dos 121 recém-nascidos estudados, $58 \%$ eram do sexo masculino. A DBP ocorreu em 55,4\% (67//2I) dos recém-nascidos estudados.

As médias da idade gestacional, peso de nascimento e escore prognóstico CRIB foram iguais nos grupos com e sem DBP na primeira e segunda semana de ventilação pulmonar mecânica; entretanto, no grupo que utilizou suporte ventilatório acima de I5 dias, o peso de nascimento e a idade gestacional foram significantemente menores no grupo com DBP (Tabela I).

A ocorrência de DBP por período de utilização de ventilação pulmonar mecânica, a razão de chance e o intervalo de confiança encontram-se na Tabela 2. 


\section{Discussão}

A ventilação pulmonar mecânica foi um grande avanço na terapêutica de pacientes críticos nas últimas três décadas. Sua utilização e o desenvolvimento de várias técnicas ventilatórias foram associadas à melhor sobrevida de pacientes portadores de insuficiência respiratória de várias etiologias, especialmente de recém-nascidos prematuros, que, devido à imaturidade pulmonar, são mais suscetíveis ao desconforto e à insuficiência respiratória.

A indicação precisa e o manejo adequado da ventilação pulmonar mecânica são armas importantes no suporte ao recémnascido prematuro. No entanto, a ventilação pulmonar mecânica não é isenta de efeitos adversos, podendo ocasionar comprometimento pulmonar, com consequêencias no desfecho desses pacientes. Nesse sentido, a redução do tempo de ventilação pulmonar mecânica foi recentemente incluída como uma das melhores práticas, visando reduzir a lesão pulmonar e prevenir a DBP em prematuros internados em unidades de cuidados intensivos neonatais ${ }^{9}$.

A principal constatação deste estudo foi a associação da ventilação pulmonar mecânica prolongada com o desenvolvimento de DBP. E o que é mais importante: a chance dessa complicação estar diretamente relacionada à utilização da ventilação pulmonar mecânica por tempo superior a sete dias, chegando a ser 16 vezes maior quando esse período ultrapassa I 5 dias. Alguns fatores podem ter influenciado este achado, como o menor peso de nascimento dos recém-nascidos. No entanto, esse fato isoladamente não o justifica, uma vez que essa diferença de peso não ocorreu entre os grupos nas duas primeiras semanas.

A prevalência de DBP varia de $20 \%$ a $40 \%{ }^{10-12}$, de acordo com a população estudada, os cuidados neonatais e os critérios diagnósticos utilizados. Na população aqui descrita, essa ocorrência foi elevada $(55,4 \%)$, sendo esse fato justificado, em parte, pela exclusão dos recém-nascidos de muito baixo peso que não necessitaram de ventilação pulmonar mecânica. Outro fator que ainda pode ter influenciado essa elevada prevalência é o critério diagnóstico utilizado. Em nosso estudo, utilizamos apenas o critério do uso contínuo de oxigênio até o $28^{\circ}$ dia de vida, enquanto que em outros estudos foram utilizados critérios variados, como a dependência de oxigênio na $36^{\mathrm{a}}$ semana de idade pós-conceptual, sugerido por Shennan ${ }^{13}$, e presença de achados radiológicos.

A utilização de oxigênio no $28^{\circ}$ dia de vida mostrou alta sensibilidade, valor preditivo positivo e negativo, para a dependência de oxigênio com 36 semanas de idade pós-conceptual nos prematuros com IG < 32 semanas e compõe a definição de DBP nessa população ${ }^{8}$, portanto, no intuito de tornar mais direta a análise dos dados, e por ser esse o ponto de corte utilizado no serviço na ocasião do estudo para definir possíveis estratégias terapêuticas, optamos por utilizar esse parâmetro único. Conforme o consenso realizado em 200 I, achados radiológicos possuem interpretações inconsistentes, não contribuindo para o diagnóstico da DBP8.

\section{ConclusÃo}

Este estudo apresenta algumas limitações inerentes aos estudos retrospectivos: a estratégia ventilatória específica não foi definida previamente, a fração inspirada de oxigênio e gasometria foram indiretamente avaliadas por meio do escore prognóstico CRIB e os fatores de confusão, como diagnósticos e terapêuticas utilizadas, não fizeram parte dos objetivos desse estudo.

Entretanto, podemos concluir que a DBP é uma complicação freqüente em recém-nascidos de muito baixo peso e está relacionada diretamente ao tempo de ventilação pulmonar mecânica, devendo a equipe que presta atendimento ao paciente de alto risco estar empenhada no desmame e extubação dos recémnascidos o mais rápido possível; de preferência, na primeira semana de vida.

Estudos prospectivos, com delineamento rigoroso, são necessários para avaliar a relação da ventilação pulmonar mecânica com DBP em nosso meio.

\section{Conflito de interesse: não há.}

\section{SUMMARY}

\section{DuRATION OF MECHANICAL Ventilation and DEVELOPMENT OF BRONCHOPULMONARY DYSPLASIA}

OBIECTIVE. Verify the association between duration of mechanical ventilation and development of bronchopulmonary dysplasia in neonates weighting at birth less than $1500 \mathrm{~g}$.

METHODS. Retrospective study conducted with neonates weighting less than $1500 \mathrm{~g}$ at birth submitted to mechanical ventilation. Neonates presenting major birth defects, transferred to other services or died before the $28^{\text {th }}$ day of life were excluded from the study. Three groups were analyzed according to duration of mechanical ventilation: I to 7 days, 8 to 14 days and more than 15 days. The chance ratio of developing bronchopulmonary dysplasia was calculated for each group.

RESULTS. From the 216 clinical histories assessed, 121 met the criteria for inclusion in the study. Mean birth weight and gestational age were $1199.8 \mathrm{~g}$ and 31.8 weeks. Of all neonates submitted to mechanical ventilation from I to 7 days, $15.5 \%$ developed bronchopulmonary dysplasia; from 8 to 14 days $60 \%$ and from more than 15 days, $88.2 \%$; chance ratios were equal to $0.16 ; 11.25$ and 16.36, respectively.

CONCLUSION. The chance of a neonate weighting less than $1500 \mathrm{~g}$ developing bronchopulmonary dysplasia was II times higher in those submitted to mechanical ventilation for up to 14 days. This chance was even higher in those ventilated for more than 15 days. That is why the nursing staff assisting high risk patients should consider the possibility of extubating neonates during their first week of life. [Rev Assoc Med Bras 2007; 53(I): 64-7]

KeY wORDS: Bronchopulmonary dysplasia. Neonates. Very low birthweight. Mechanical ventilation.

\section{REFERÊNCIAS}

I. Avery ME, Tooley WH, Keller JB, Hurd SS, Bryan H, Cotton RB, et al. Is chronic lung disease in low birthweight infants preventable? A survey of eight centers. Pediatrics. 1987;79:26-30. 
2. Dreyfuss D, Saumon G. Ventilator-induced lung injury: lessons from experimental studies. Am J Resp Crit Care Med. 1998; 157:294-323.

3. Coalson JJ, Winter VT, Siler-Khodr T, Yoder BA. Neonatal chronic lung disease in extremely immature baboons. Am J Respir Crit Care Med. 1999; 160: 1333-46.

4. Albertine KH, Jones GP, Starcher BC, Bohnsack JF, Davis PL, Cho SC, et al. Chronic lung injury in preterm lambs. Disordered respiratory tract development. Am J Respir Crit Care Med. 1999;159:945-58.

5. Vassalo JC, Sasbón JS. Lesión pulmonar inducida por la ventilacion pulmonar mecânica. In: Carvalho, WB, Jiménez HJ, Sasbón JS. Ventilación pulmonar mecânica en pediatria. São Paulo: Atheneu; 200I . p.213-25.

6. Leão LL, Aguiar MJB. Síndromes dismórficas com alteração do sistema nervoso central. In: Fonseca LF, Pianetti G, Xavier CC. Compêndio de Neurologia Infantil. Rio de Janeiro: Medsi; 2002. p.669-83.

7. Borrelli VLM. Escore Prognóstico. In: Fázio Jr João, Carvalho MF, Nogueira PRC, Carvalho WB. Cuidados intensivos no período neonatal. São Paulo: Sarvier; 1999. p.30- I.

8. Jobe AH, Bancalari E. Bronchopulmonary Dysplasia. AmJ Respir Crit Care Med. 2001; 163:1723-9.

9. Sharek PJ, Baker R, Litman F, Kaempf J, Burch K, Schwarz E, et al. Evaluation and development of potentially better practices to prevent chronic lung disease and reduce lung injury in neonates. Pediatrics. 2003; I I I:426-31

10. Manktelow BN, Draper ES, Annamalai S, Field D. Factors affecting the incidence of chronic lung disease of prematurity in 1987, 1992, and 1997. Arch Dis Child Fetal Neonatal Ed. 200 I;85:33-5.

I I. Verhagen AA, Keli SO, Meulen GN van der, Wiersma H, Arias M, Angelista $\mathbb{I R}$, et al. Surfactant treatment in premature infants with Respiratory Distress Syndrome in Curaçao. West Indian Med. 200 I;50:1 17-22.

12. Cunha GS, Mezzacapa Filho F, Ribeiro JD. Fatores maternos e neonatais na incidência de displasia broncopulmonar em recém-nascidos de muito baixo peso. J Pediat (Rio J). 2003;79:550-6.

13. Shennan AT, Dunn MS, Ohlsson A, Lennox K, Hoskins EM. Abnormal pulmonary outcomes in premature infants: Prediction from oxygen requirement in the neonatal period. Pediatrics. 1988;82:527-32.

Artigo recebido: I / /02/06

Aceito para publicação: 30/10/06 